\title{
Autophagic and lysosomal defects in human tauopathies: analysis of post- mortem brain from patients with familial Alzheimer disease, corticobasal degeneration and progressive supranuclear palsy
}

Antonio Piras', Ludovic Collin², Fiona Grüninger², Caroline Graff ${ }^{1,3}$ and Annica Rönnbäck ${ }^{1 *}$

\begin{abstract}
Introduction: The accumulation of insoluble proteins within neurons and glia cells is a pathological hallmark of several neurodegenerative diseases. Abnormal aggregation of the microtubule-associated protein tau characterizes the neuropathology of tauopathies, such as Alzheimer disease (AD), corticobasal degeneration (CBD) and progressive supranuclear palsy (PSP). An impairment of the lysosomal degradation pathway called macroautophagy, hereafter referred to as autophagy, could contribute to the accumulation of aggregated proteins. The role of autophagy in neurodegeneration has been intensively studied in the context of AD but there are few studies in other tauopathies and it is not known if defects in autophagy is a general feature of tauopathies. In the present study, we analysed autophagic and lysosomal markers in human post-mortem brain samples from patients with early-onset familial AD (FAD) with the APP Swedish mutation (APPswe), CBD and PSP and control individuals.

Results: FAD, CBD and PSP patients displayed an increase in LC3-positive vesicles in frontal cortex, indicating an accumulation of autophagic vesicles. Moreover, using double-immunohistochemistry and in situ proximity ligation assay, we observed colocalization of hyperphosphorylated tau with the autophagy marker LC3 in FAD, CBD and PSP patients but not in control individuals. Increased levels of the lysosomal marker LAMP1 was detected in FAD and CBD, and in addition Cathepsin D was diffusely spread in the cytoplasm in all tauopathies suggesting an impaired lysosomal integrity.
\end{abstract}

Conclusion: Taken together, our results indicate an accumulation of autophagic and lysosomal markers in human brain tissue from patients with primary tauopathies (CBD and PSP) as well as FAD, suggesting a defect of the autophagosome-lysosome pathway that may contribute to the development of tau pathology.

Keywords: Autophagy, Alzheimer disease, Tauopathy, Dementia, Hyperphosphorylated tau

\footnotetext{
* Correspondence: annica.ronnback@ki.se

${ }^{1}$ Karolinska Institutet, Department NVS, Center for Alzheimer Research,

Division for Neurogeriatrics, Karolinska Institutet, SE-141 57 Huddinge,

Sweden

Full list of author information is available at the end of the article
} 


\section{Introduction}

Abnormal intracellular aggregation and accumulation of the microtubule-associated protein tau is a common feature of many neurodegenerative disorders, including Alzheimer disease (AD), frontotemporal lobar degeneration, Pick's disease, argyrophilic grain disease, corticobasal degeneration (CBD) and progressive supranuclear palsy (PSP) [3]. Collectively, these neurodegenerative disorders are referred to as tauopathies. Under normal conditions, tau is predominantly expressed in neuronal axons where its main function is to promote microtubule assembly and stabilization, which is important for maintaining axonal transport and neuronal integrity $[45,60,69]$. The molecular mechanisms leading to intracellular tau aggregation in AD and other tauopathies are not fully understood, but abnormal posttranslational modifications, such as hyperphosphorylation and acetylation [20,47], and impaired degradation of tau [14] have been suggested.

Two major proteolytic systems contribute to tau degradation, the ubiquitin-proteasome system (UPS) and the autophagy-lysosome system [50, 52, 67]. The role of each of these pathways to the turnover of tau is an area of significant interest [16, 32, 38, 40, 66, 67]. The major lysosomal degradative pathway in eukaryotic cells, macroautophagy (hereafter referred to as autophagy), is responsible for degrading long-lived or aggregated proteins and is the principal mechanism for turning over cellular material too large to be degraded by the UPS $[29,75,76]$. Autophagy is a highly regulated process that involves the sequestration of cytoplasmic cargo, such as aggregated proteins and damaged organelles, within double-membrane vesicles called autophagosomes which are typically labelled by the microtubule-associated protein 1A/1B-light chain 3 (LC3) $[23,29,30,72,76]$. In order to achieve degradation of aggregated proteins in the autophagy-lysosome system, there must be a successful fusion of the autophagosomes with the lysosomes [17, 18, 29, 36, 73]. Functional autophagy is crucial for neuronal physiology and loss of autophagy in the central nervous system, for example by inactivation of key autophagy genes such as autophagy-related (Atg) proteins 5 (Atg5) or 7 (Atg7) leads to neurodegeneration [22, 31]. In Atg7 knockout mouse brains, there is a significant accumulation of hyperphosphorylated tau suggesting a role of autophagy in the clearance of pathological tau in adult neurons [25]. Furthermore, increased accumulation of autophagic vesicles has been reported in human postmortem AD brains and in mouse models of tauopathy [41, 54, 77]. Constitutive overexpression of $\mathrm{mTor}$, (mammalian Target of rapamycin), a key negative regulator of the autophagic pathway, prevents activation of the autophagy pathway and increases the levels of hyperphosphorylated tau in a cell model of tauopathy [65]. Conversely, autophagy enhancers like rapamycin (an mTor inhibitor) or trehalose (an mTor-independent autophagy activator) can promote the degradation of insoluble tau in mouse models of tauopathy $[9,56,61]$. Finally, post-translational modifications of tau can interfere with and impair the clearance mechanisms. For example, phosphorylation of tau at serine 422 (Tau/ pS422) prevents tau cleavage by caspase-3 at aspartic acid 421 (D421), precluding tau degradation by the autophagy-lysosome system [21]. Taken together these observations suggest that the autophagy-lysosome pathway plays an important role in the clearance of hyperphosphorylated tau. The majority of studies on human neurodegenerative disease and autophagy have included patients with Alzheimer disease where both $A \beta$ and tau aggregations are key features (reviewed in $[43,55]$ ), and only few studies have focused on other human tauopathies $[19,71]$. Thus, in order to address the contribution of the autophagy-lysosomal system in different tauopathies, we studied human post-mortem brain tissue from patients with both tau and $A \beta$ pathology [familial AD (FAD) cases with the Swedish doublemutation in the amyloid precursor protein (APPswe)] as well as brain tissue from patients with a primary tauopathies in the absence of significant amyloid pathology (CBD and PSP). In agreement with previous studies of sporadic AD cases [42, 51, 54], we found an accumulation of markers of the autophagy-lysosomal pathway in $\mathrm{AD}$ patients with the familial APPswe mutation. In addition, we showed that the autophagy-lysosomal system is impaired in patients with primary tauopathies suggesting that autophagic defects are a common feature of human tauopathies.

\section{Material and methods Brain samples}

Human post-mortem brain tissue samples from frontal cortex were obtained from the Brain Bank at Karolinska Institutet. Three patients with early onset familial AD (FAD) caused by the Swedish amyloid precursor protein gene double-mutation KM670/671NL (APPswe), four patients with CBD and three patients with PSP as well as brain tissue from six control subjects (absence of neurodegenerative disease) were included (Table 1).

\section{Immunohistochemistry}

Immunohistochemical staining was performed on $5 \mu \mathrm{m}$ sections from formalin fixed paraffin embedded (FFPE) frontal cortex of post-mortem brains. The sections were deparaffinised and hydrated through xylene and graded alcohol series. The sections were autoclaved with antigen retrieval buffer (DV2004, DIVA Decloaker, Biocare Medical) for $30 \mathrm{~min}$ at $110{ }^{\circ} \mathrm{C}$ (Decloaking Chamber NxGen, 
Table 1 Human brain samples

\begin{tabular}{|c|c|c|c|c|c|c|}
\hline Case no & Age at death (years) & Gender (M/F) & Age at onset (years) & PMI (hours/days) & Brain weight (g) & Analysis \\
\hline Ctrl_1 & 70 & M & - & $18 \mathrm{~h}$ & 1482 & $\mathrm{IHC}, \mathrm{IF}$ \\
\hline Ctrl_2 & 64 & $\mathrm{~F}$ & - & $5 \mathrm{~h}$ & 1204 & $\mathrm{IHC}, \mathrm{IF}, \mathrm{PLA}, \mathrm{WB}$ \\
\hline Ctrl_3 & 84 & $\mathrm{~F}$ & - & $96 \mathrm{~h}$ & 1210 & $\mathrm{IHC}, \mathrm{IF}, \mathrm{PLA}$ \\
\hline Ctrl_4 & 59 & M & - & na & 1390 & $\mathrm{IHC}, \mathrm{IF}, \mathrm{PLA}$ \\
\hline Ctrl_5 & 82 & $\mathrm{~F}$ & - & $9 \mathrm{~h}$ & 1200 & WB \\
\hline Ctrl_6 & 80 & M & - & $16 \mathrm{~h}$ & 1200 & WB \\
\hline APPswe_1 & 62 & M & 53 & $40 \mathrm{~h}$ & 1024 & $\mathrm{IHC}, \mathrm{IF}, \mathrm{PLA}, \mathrm{WB}$ \\
\hline APPswe_2 & 66 & M & 61 & $24 \mathrm{~h}$ & 1140 & $\mathrm{IHC}, \mathrm{IF}, \mathrm{PLA}, \mathrm{WB}$ \\
\hline APPswe_3 & 56 & M & 44 & $24 \mathrm{~h}$ & 1161 & $\| H C, I F, P L A, W B$ \\
\hline CBD_1 & 80 & $\mathrm{~F}$ & 74 & 8 days & 1075 & $\mathrm{IHC}, \mathrm{IF}, \mathrm{PLA}, \mathrm{WB}$ \\
\hline CBD_2 & 69 & M & 62 & $66 \mathrm{~h}$ & $\sim 1000$ & IHC, IF, PLA \\
\hline CBD_3 & 70 & M & 64 & $24 \mathrm{~h}$ & 930 & $\mathrm{IHC}, \mathrm{IF}, \mathrm{PLA}$ \\
\hline $\mathrm{CBD}_{-} 4$ & 67 & $\mathrm{~F}$ & 59 & $48 \mathrm{~h}$ & 1045 & WB \\
\hline PSP_1 & 66 & $\mathrm{~F}$ & 59 & $144 \mathrm{~h}$ & 1490 & $\mathrm{IHC}, \mathrm{IF}, \mathrm{PLA}$ \\
\hline PSP_2 & 71 & $\mathrm{~F}$ & 63 & na & 1121 & $\mathrm{IHC}, \mathrm{IF}, \mathrm{PLA}$ \\
\hline PSP_3 & 68 & M & $<66$ & na & 1350 & $\mathrm{IHC}, \mathrm{IF}, \mathrm{PLA}$ \\
\hline
\end{tabular}

PMI post-mortem interval, time from death until autopsy, IHC immunohistochemistry, IF immunofluorescence, PLA proximity ligation assay, WB western blot, na not available

Biocare Medical). After the temperature decreased to room temperature (RT), sections were washed with water for $5 \mathrm{~min}$ and then in Tris-Buffered Saline (TBS) $+0.05 \%$ Tween 20 (TBS-T) (91414, Sigma-Aldrich). The sections were incubated in Peroxidase block solution (K4007, Dako) $5 \mathrm{~min}$ at RT to quench the endogenous peroxidase activity. Sections were then incubated with primary antibodies (Table 2) diluted in Antibody Diluent (S3022, Dako) for $45 \mathrm{~min}$ at RT, followed by $30 \mathrm{~min}$ incubation with EnVision Mouse (K4007, Dako) at RT. The immunoreactions were visualized with DAB (K4011, Dako). All sections were counterstained with haematoxylin for $30 \mathrm{~s}$ and washed in tap water. The sections were washed thoroughly in TBS-T between each step. Dehydration was performed in increasing concentrations of ethanol, cleared in xylene and mounted with DPX mountant $(360294 \mathrm{H}$,
VWR). For every experiment, tissue sections from a control or a tauopathy-patient were incubated without the primary antibody to be used as a negative control.

For immunofluorescence staining, all sections were processed under the same standardized conditions, following the method described above with minor modifications. After deparaffinization and antigen retrieval, the sections were blocked with Background Punisher (BP974, Biocare Medical) for $10 \mathrm{~min}$ at RT followed by washing and incubation with primary antibodies (Table 2) in TBS$\mathrm{T}$ (overnight $4{ }^{\circ} \mathrm{C}$, humid chamber). After washing in TBS-T, sections were incubated ( $1 \mathrm{~h}$ at $\mathrm{RT})$ with appropriate secondary antibodies [anti-mouse and anti-rabbit IgG $(\mathrm{H}+\mathrm{L})$ ] conjugated to Alexa Fluor 546 (A-11003, Invitrogen) or Alexa Fluor 488 (A-11008, Invitrogen) at a concentration of 1:500 in TBS-T. After washing in

Table 2 List of primary antibodies

\begin{tabular}{|c|c|c|c|c|c|c|}
\hline Antigen & Supply & Reference & Clonality & Host & Dilution & Analysis \\
\hline AT8 & Thermo Scientific & MN1020 & Monoclonal & Mouse & $1: 1000$ & $\overline{I H C, P L A}$ \\
\hline Tau/pS422 & Roche & - & Monoclonal & Mouse & $1: 1000$ & IF \\
\hline LC3 & Novus Biologicals & NB100-2331 & Polyclonal & Rabbit & $1: 200$ & IF PLA \\
\hline p62/SQSTM1 & Santa Cruz & sc- -25575 & Polyclonal & Rabbit & $1: 50$ & IF \\
\hline LAMP1 & Santa Cruz & sc-20011 & Monoclonal & Mouse & $1: 200$ & WB \\
\hline Cathepsin D (Cat D) & Sigma-Aldrich & HPA003001 & Polyclonal & Rabbit & $1: 500$ & IF \\
\hline Cathepsin D (Cat D) & Abcam & Ab6313 & Monoclonal & Mouse & $1: 500$ & IF \\
\hline GAPDH & Enzo & ID4 & Monoclonal & Mouse & $1: 2000$ & WB \\
\hline
\end{tabular}

IHC immunohistochemistry, IF immunofluorescence, PLA proximity ligation assay, WB western blot 
TBS-T $(3 \times 10 \mathrm{~min})$ slides were incubated with (or without) Sudan Black B (199664, Sigma-Aldrich), 5 min at $\mathrm{RT}$ to reduce autofluorescence. Sections were then washed in TBS-T and incubated with DAPI for $5 \mathrm{~min}$ (D9564, Sigma-Aldrich). After washing in TBS-T, coverslips were mounted with Vectashield Hard Set (H1200, Vector Laboratories) and the slides were stored at $4{ }^{\circ} \mathrm{C}$. Sections from controls and patients were also incubated without primary antibody and used as negative control. Sections were examined using a laser scanning confocal microscope (LSM 510 META, ZEISS), and images were acquired using the same settings (laser intensity, detector gain and amplifier offset).

\section{In situ Proximity Ligation Assay (PLA)}

To specifically detect protein interaction (when the proteins are in close proximity $<40 \mathrm{~nm}$ ), PLA analyses were performed on section from FFPE frontal cortex of human brain from three patients of each group (Table 1). Deparaffinization and antigen retrieval were performed as described above. In situ PLA was performed according to the OLINK Bioscience instructions. To reduce non-specific signals, the sections were incubated with Blocking solution (82007, OLINK Bioscience) for $30 \mathrm{~min}$ at $37{ }^{\circ} \mathrm{C}$. Sections were then incubated overnight at $4{ }^{\circ} \mathrm{C}$ with two primary antibodies (Table 2 ) in antibody diluent solution (82008, OLINK Bioscience) (80-100 $\mu \mathrm{l} /$ section). For each patient, two negative controls were performed by omitting one of the two primary antibodies (negative control 1 and negative control 2). After washing with TBS-T, sections were incubated with in situ PLA DNA-probes anti-rabbit PLUS and anti-mouse MINUS (82002 and 82004, OLINK, Bioscience) for $1 \mathrm{~h}$ at $37^{\circ} \mathrm{C}$. Ligation solution was added for $30 \mathrm{~min}$ at $37^{\circ} \mathrm{C}$, and washed twice with washing Buffer A (82047, OLINK, Bioscience). The amplification solution (92013, Detection reagents Far Red, OLINK Bioscience) was added for $100 \mathrm{~min}$ at $37^{\circ} \mathrm{C}$. Following incubation the sections were washed in Buffer $B$ (82048, OLINK, Bioscience). The sections were allowed to dry and mounted with Vectashield Hard Set mounting medium with DAPI (H-1200, Vector Laboratories). Images from at least ten different fields of the frontal cortex for each sample were acquired using a laser scanning confocal microscope (LSM 510 Meta, ZEISS). Then, the images were analyzed with DUOLINK Image Tool software (OLINK Bioscience), which automatically counts the number of fluorescent signals (dots) per field. The signal was calculated as Dots $=$ Dots $_{\mathrm{PLA}}-\left(\right.$ Dots $_{\text {neg1 }}+$ Dots $\left._{\text {neg2 }}\right)$, where the Dots PLA is the number of dots of in situ PLA and Dots neg1 $_{\text {and Dots }}$ neg2 are respectively the dots obtained from negative control 1 [only primary antibody 1 , anti-tau clone AT8 (pS202/T205)] and negative control 2 (only primary antibody 2, LC3) [62]. Data were expressed as mean value \pm Standard Deviation (S.D.) and ANOVA, followed by the Dunnett post-hoc test, was used for statistical analysis (IBM SPSS Software). Significance levels of * $p<0.05, * * 0<0.01, * * * 0<0.001$ were used.

\section{Western blotting}

Frozen tissue from frontal cortex $(\sim 145 \mathrm{mg} / \mathrm{sample})$ of human brains from FAD $(n=3), \operatorname{CBD}(n=2)$ and Control $(n=3)$ (Table 1$)$ were homogenized in RIPA buffer $(50 \mathrm{mM}$ Tris $\mathrm{pH} 7.5 ; 150 \mathrm{mM} \mathrm{NaCl} ; 0.5 \%$ sodium deoxycholate; $0.1 \%$ SDS; 1 \% NP40) containing Benzonase Nuclease (70664-3, Millipore, 1:1000), Phosphatase and Protease inhibitor cocktails (P8340 and P0044, Sigma-Aldrich, 1:100) on ice. Samples were centrifuged at 7000xg for $10 \mathrm{~min}$ at $4{ }^{\circ} \mathrm{C}$. Supernatants were stored at $-20{ }^{\circ} \mathrm{C}$.

After determination of the protein content by BCA protein assay (23227, Thermo Fischer Scientific), homogenates (30-50 $\mu \mathrm{g}$ total protein) were separated on NuPAGE $^{\oplus} 12 \%$ Bis-Tris Gels, 1.0 mm, 10 wells (NP0341, Life Technologies) by electrophoreses in running buffer MOPS [3-( $N$-morpholino) propanelsulfonic acid] (NP0001, Life Technologies) and blotted onto iBlot Gel Transfer Stacks Nitrocellulose (IB301001, Life Technologies). The membranes were blocked in $5 \%$ non-fat milk (70166, Sigma-Aldrich) in TBS-T. Membranes were incubated overnight at $4{ }^{\circ} \mathrm{C}$ with primary antibodies (see Table 2), washed in TBS-T and then incubated with secondary species-specific antibodies (NA934 anti-rabbit, NA931 anti-mouse, GE Healthcare) in TBS-T + $5 \%$ non-fat milk for $1 \mathrm{~h}$ at RT. Blots were visualized with enhanced chemiluminescence reagent (34076, Pierce). Images were obtained with a FujiFilm LAS-3000 camera and semiquantitative analysis was performed using Image J software. Data were expressed as mean intensity of an arbitrary unit (AU) \pm S.D. and were obtained from the average of at least 3 replications of the experiment. For quantification of immunoblots, protein levels were normalized to GAPDH. ANOVA, followed by the Dunnett post-hoc test, was used to compute statistical significance and significance levels of $* p<$ $0.05,{ }^{* *} p<0.01,{ }^{* * *} p<0.001$ were used.

\section{Results}

Characterization of tau pathology in frontal cortex from patients diagnosed with different tauopathies

Human post-mortem brain tissue from cases with earlyonset FAD, CBD, PSP and control individuals without neurodegeneration was analysed by immunohistochemistry to determine the presence of hyperphosphorylated tau using two different phospho-tau antibodies: clone AT8 (pS202/T205, Fig. 1) and Tau/pS422 (Additional file 1: Figure S1). Patients carrying the APPswe mutation showed extensive tau immunoreactivity in neurofibrillary tangles (Fig. 1a, large arrow), pretangles (defined by diffuse cytoplasmic tau immunoreactivity, Fig. 1a, asterisk), dystrophic 


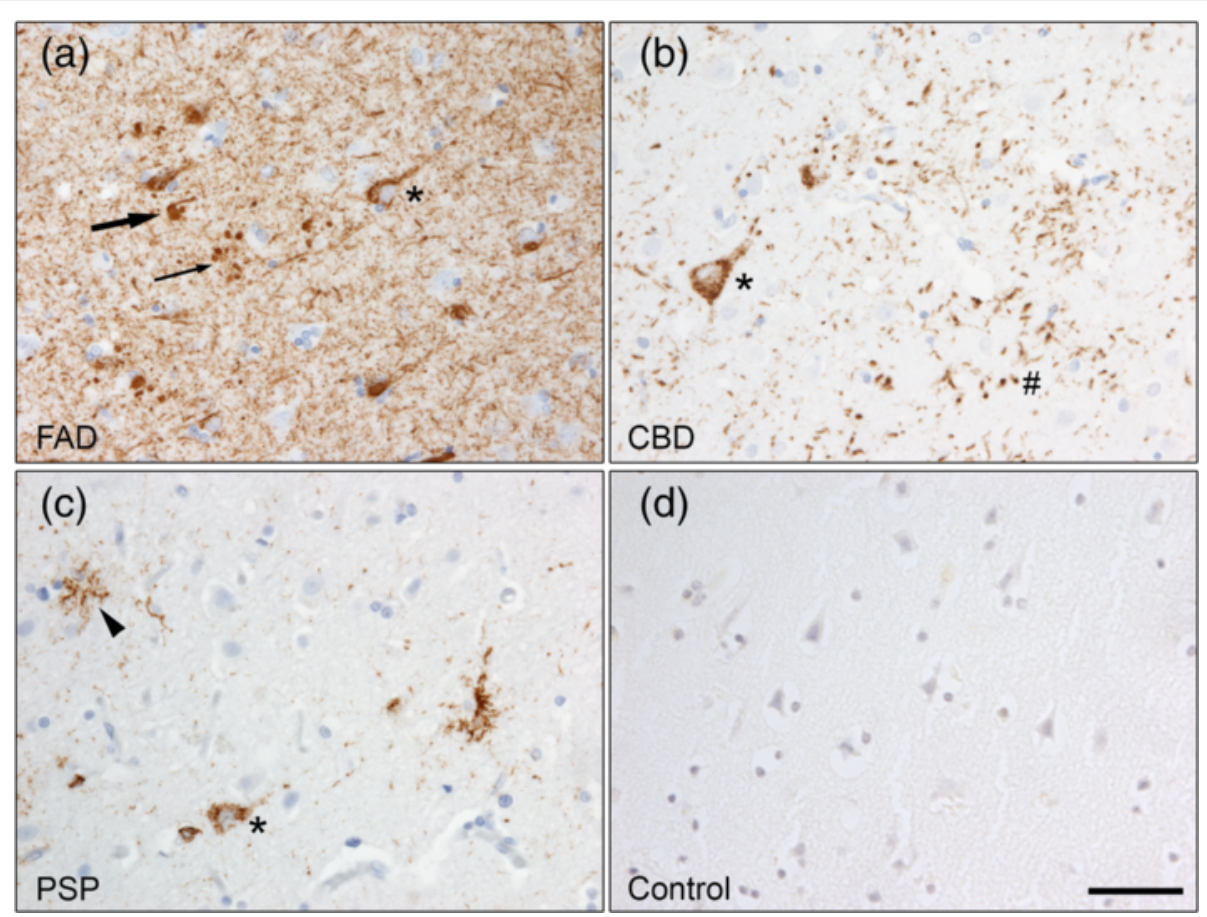

Fig. 1 Tau pathology in frontal cortex as showed with the AT8 antibody. a APPswe mutation carriers show AT8 immunoreactivity in neurofibrillary tangles (arrow), pretangles (asterisk), neuropil threads and dystrophic neurites around amyloid plaques (small arrow). b AT8 immunohistochemistry revealed diffuse cytoplasmic tau immunoreactivity in neurons (pretangles, asterisk) and astrocytic plaques (\#) in CBD patients, while c PSP patients show AT8 immunoreactivity in pretangles (asterisk) and tufted astrocytes (arrowhead). d No AT8 immunoreactivity was detected in control individuals. Scale bar: $50 \mu \mathrm{m}$

neurites around amyloid plaques (Fig. 1a, small arrow) and neuropil threads. All CBD cases showed hyperphosphorylated tau in neuronal pretangles (Fig. 1b, asterisk), astrocytic plaques (Fig. 1b, \#), threads in grey and white matter and oligodendroglial coiled bodies (data not shown). Tauimmunoreactive pretangle neurons (Fig. 1c, asterisk), tufted astrocytes (Fig. 1c, arrowhead) and coiled bodies in oligodendroglia (data not shown) were present in frontal cortex of the PSP cases. In contrast, no tau immunoreactivity was detected in the control individuals (Fig. 1d). Our data confirmed that hyperphosphorylated tau accumulates in the frontal cortex of all three patient groups.

\section{Accumulation of p62/SQSTM1 and colocalization with hyperphosphorylated tau in frontal cortex from cases with tauopathies}

The polyubiquitin-binding protein p62/SQSTM1 is necessary to target protein aggregates for degradation via autophagy $[6,57]$, and/or the ubiquitin-proteasome system $[2,4,63]$. To investigate whether the protein clearance was defective in patients with tauopathies, we analysed the p62/SQSTM1 protein by immunostaining. Only rare p62/SQSTM1 inclusions were detected in control brain sections (Fig. 2a, green), while we observed an intense p62/SQSTM1 immunostaining in FAD, CBD and PSP (Fig. 2b-d, green), showing that p62/SQSTM1 accumulates in patients with tauopathies. Binding of p62/SQSTM1 to hyperphosphorylated tau is necessary for the clearance of tau as reported in p62knockout mice that showed an accumulation of hyperphosphorylated tau and neurofibrillary tangles [2]. Therefore we investigated the colocalization of p62/ SQSTM1 and phospho-tau (Tau/pS422, Fig. 2f-h). We found that p62/SQSTM1 colocalized with hyperphosphorylated tau in neuronal cell bodies (arrowheads in Fig. 2 j, n) and in neuropil threads (asterisks in Fig. 2j, m) in FAD patients. In CBD patients, an extensive colocalization of p62/SQSTM1 and hyperphosphorylated tau was found mainly in threads (asterisks in Fig. $2 \mathrm{k}$ and high magnification Fig. 2o). Finally, in PSP patients we found colocalization of p62/SQSTM1 and hyperphosphorylated tau both in threads (asterisks in Fig. 2l, p) and in cell bodies (arrowhead in Fig. 2l, p), similar to what we observed in FAD patients. Altogether, the accumulation of p62/SQSTM1 and its colocalization with hyperphorylated tau suggest defective protein clearance in tauopathy patients, which is in accordance with previous studies $[33,34]$. 


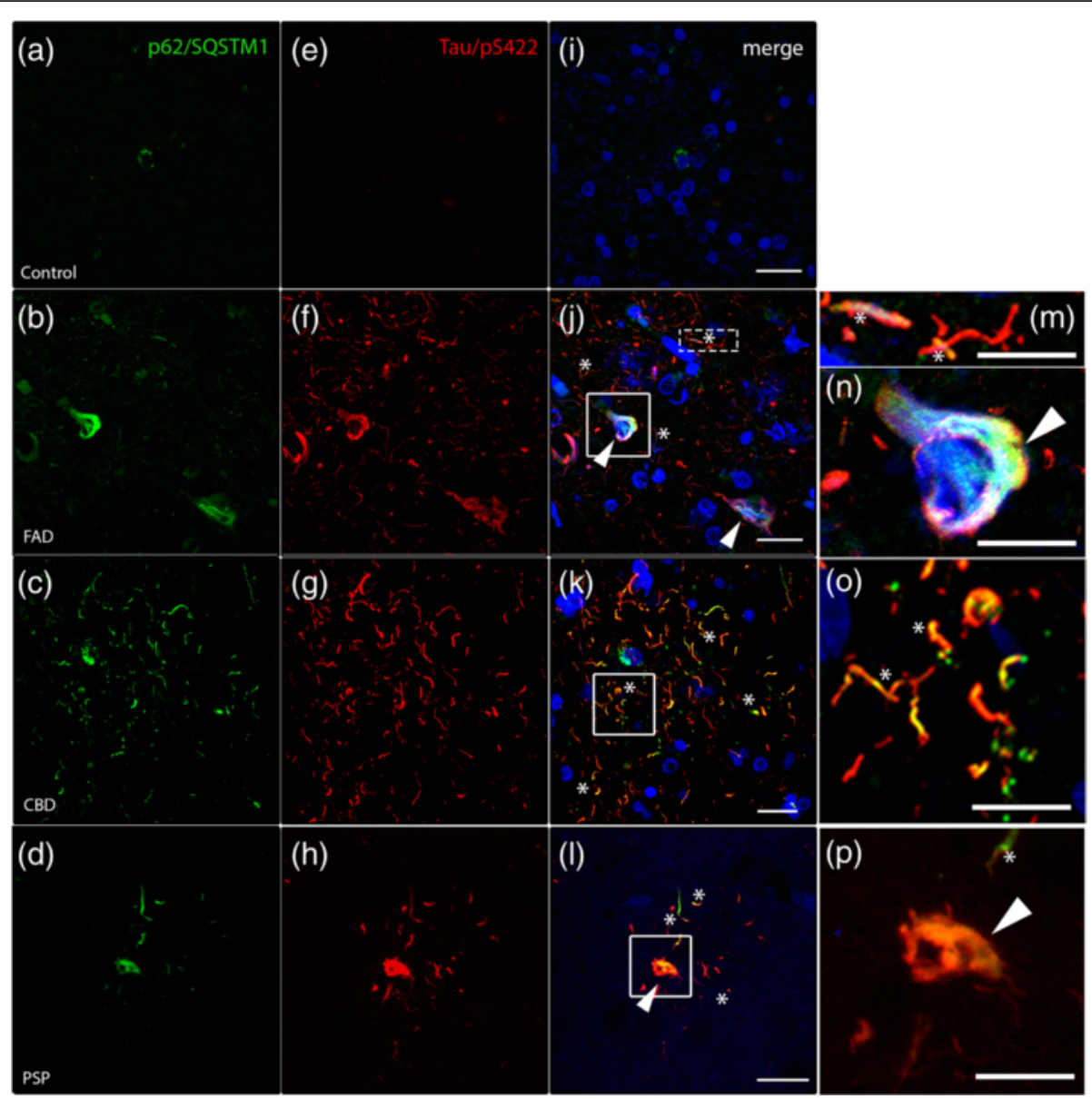

Fig. 2 The autophagic marker p62/SQSTM1 accumulates and colocalizes with hyperphosphorylated tau in human tauopathies. Double immunohistochemistry against p62/SQSTM1 (green), hyperphosphorylated tau (Tau/pS422, red) and DAPI (blue) are shown by confocal analysis. a In control individuals, p62/SQSTM1-positive inclusions are only rarely observed and (e, i) no Tau/pS422 immunoreactivity were detected. b-h Strong immunoreactivity of p62/SQSTM1 (b-d) and Tau/pS422 (f-h) in post-mortem brain sections from FAD, CBD and PSP patients. $\mathbf{j}-\mathbf{I}$ Merged pictures show colocalization between p62/SQSTM1 and Tau/pS422. $\mathbf{j}$ In FAD patients, colocalization is shown in neuronal threads (asterisks and high magnification $\mathbf{m}$ ) and close to the nucleus (arrowheads and high magnification $\mathbf{n}$ ). $\mathbf{k}, \mathbf{o}$ In CBD patients, asterisks indicate colocalization in threads. I, p In PSP brains, colocalization is detected in threads (asterisks) and in some cell bodies (arrowheads). Scale: $20 \mu \mathrm{m}$ (10 $\mu \mathrm{m}$ insert)

Accumulation of LC3-positive structures in frontal cortex from patients with taoupathies and colocalization with hyperphosphorylated tau

Several studies have shown that hyperphoshorylated tau can be cleared by the autophagy pathway [14, 16, 24, 66] and accumulation of pathological hyperphosphorylated tau and p62/SQSTM1 could result from defective autophagylysosomal clearance [35, 68]. To investigate whether the autophagy system is impaired in human tauopathies, we analysed the expression of LC3, a well-established autophagosome marker [29], by immunostaining of frontal cortex sections from controls and patients. In control individuals, we observed diffuse LC3 immunostaining in the cytoplasm and only rarely detected LC3-positive puncta (Fig. 3e, i, m). In contrast, in FAD patients (Fig. 3f, j, n), we observed an increase in LC3-positive puncta in the perinuclear cytoplasm (arrowheads in Fig. 3j, n) and in threads (asterisks in Fig. 3j). Interestingly, we also observed a marked increase of LC3positive puncta in the cytoplasm of cells in the frontal cortex of CBD (Fig. 3g, k, o, arrowheads) and PSP patients (Fig. 3h, l, p, arrowheads). Altogether, these results suggest that accumulation of autophagic vesicles is a common feature in patients with tauopathies. In addition to the LC3-positive puncta in the perinuclear cytoplasm, we observed strong LC3 immunoreactivity in places distant from any clearly visible soma in all three patient groups but not in controls (Fig. 3).

Next we investigated if hyperphosphorylated tau colocalized with LC3 in patients with tauopathies. In FAD patients, colocalization between hyperphosphorylated tau (Tau/pS422) and LC3 was found in tangle-like 


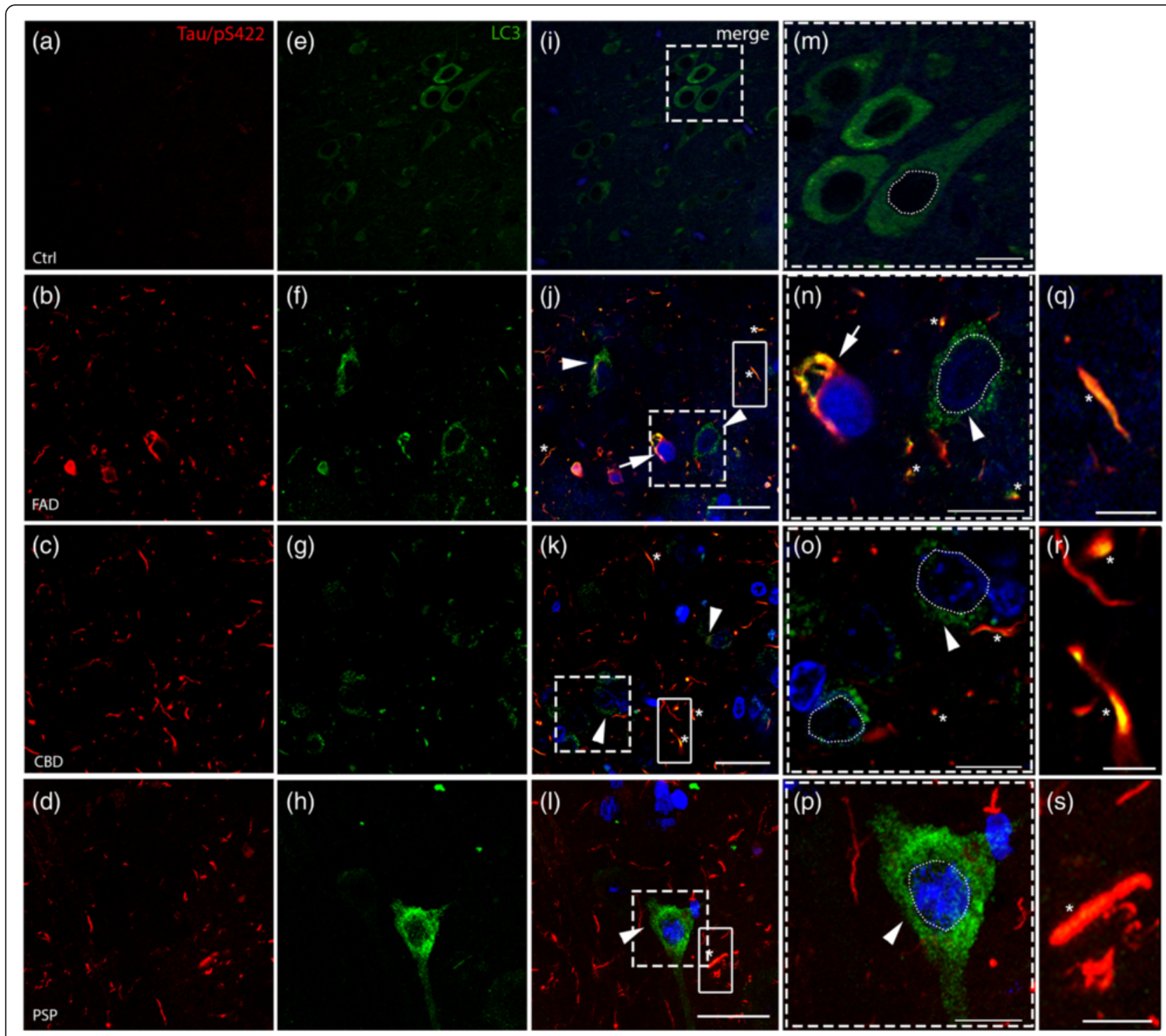

Fig. 3 Accumulation of autophagic marker (LC3) and colocalization with hyperphosphorylated tau (Tau/pS422). Double immunofluorescence analysis on post-mortem tissues against (a-d) Tau/pS422 (red) and (e-h) LC3 (green). j-p Nuclei are stained with DAPI (blue, outlined with a white dashed line) in the merged pictures. e In control samples, LC3 staining is diffuse in the cytoplasm and LC3-positive dots are rarely observed (high magnification in ( $\mathbf{m}$ ) of the boxed area in $\mathbf{i}) \mathbf{j}$ - $\mathbf{l}$ Arrowheads (and high magnifications, $\mathbf{n}-\mathbf{p}$ ) indicate cells showing accumulation of LC3 positive puncta in the perinuclear cytoplasm in FAD, CBD and PSP patients. Asterisks show colocalization in threads. In addition, FAD shows colocalization in tangle-like structures (j, $\mathbf{n}$, arrows, see also SF. 2). q-s High magnification of the boxed areas in $\mathbf{j}-\mathbf{I}$ showing colocalization of Tau/pS422 and LC3 in threads, respectively in FAD, CBD and PSP. Scale bars indicate $50 \mu \mathrm{m}$ (i) and $25 \mu \mathrm{m}$ (j-l); high magnification $10 \mu \mathrm{m}(\mathbf{m}-\mathbf{p})$ and $5 \mu \mathrm{m}(\mathbf{q}-\mathbf{s})$

structures (arrows in Fig. 3j, n; see also Additional file 2: Figure S2) and in neuropil threads (asterisks in Fig. 3j and high magnification Fig. 3n, q). CBD and PSP patients showed colocalization of hyperphosphorylated tau and LC3 mainly in threads (asterisks in Fig. 3k, 1 and high magnifications in Fig. 3o, r, s), although we cannot exclude that this is a result of the low levels of hyperphosphorylated tau in neuronal soma in CBD and PSP patients.

To confirm the colocalization between hyperphosphorylated tau and LC3, we performed in situ proximity ligation assay (PLA) analysis, a technique to detect and quantify protein-protein interaction with high specificity and sensitivity [64]. We detected significant PLA signals for hyperphosphorylated tau (AT8) and LC3 in the frontal cortex of FAD, CBD and PSP cases (Fig. 4b-d), while no colocalization signal was observed in control individuals (Fig. 4a). The PLA signal was often observed in cell bodies (Fig. 4f, asterisk) and in the neuropil threads (arrowhead) in FAD patients. In contrast, CBD and PSP patients rarely showed PLA signal in the 

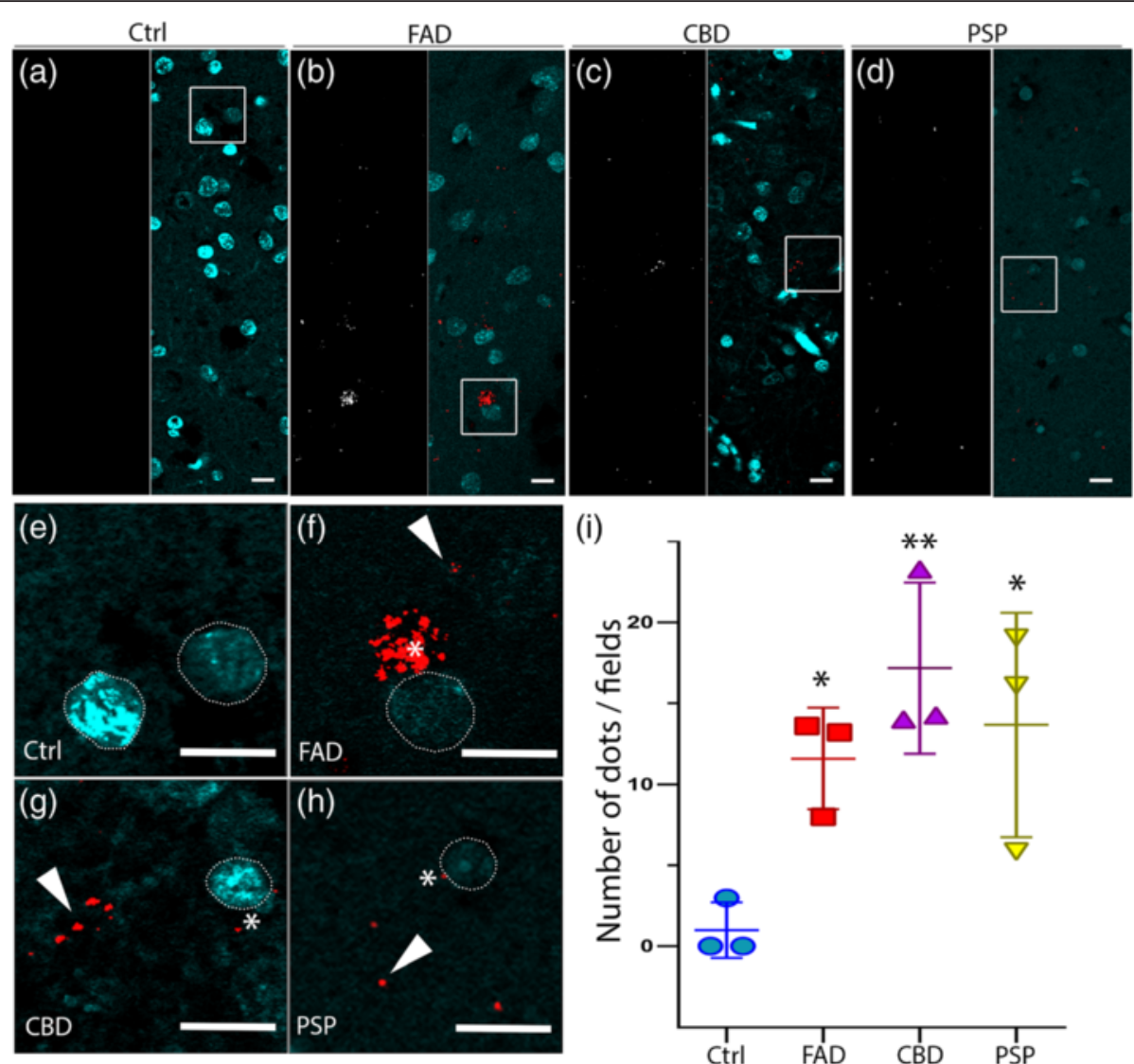

Fig. 4 In situ PLA on human brain tissue confirms colocalization between hyperphosphorylated tau (AT8) and LC3. a-d The white signal shows the PLA dots in healthy controls and patients, respectively. In merged pictures, PLA staining (red) and DAPI (blue) are shown in FAD, CBD and PSP. PLA dots are rarely observed in control individuals. $\mathbf{e}-\mathbf{h}$ High magnification of the boxed area from the merged pictures. Asterisks show PLA signal close to the nucleus (white dashed line), and arrowheads indicate PLA signal distant from clearly visible cell soma in FAD, CBD and PSP patients. Scale: $10 \mu \mathrm{m}$. $\mathbf{i}$ In the graphs PLA signal (number of dots/field) are indicated as Mean value \pm S.D. from healthy controls and patients

perinuclear region (Fig. 4g, h, asterisks), and mainly further away from cell nuclei (Fig. 4g, h, arrowheads), consistent with the double-immunofluorescence data. Quantification of the PLA signal (Fig. 4i) showed significantly more colocalization of hyperphosphorylated tau and LC3 in FAD (11.5 \pm 2.2 dots/field), CBD (17.2 \pm 5.3 dots/field) and PSP $(13.9 \pm 6.9$ dots/field) compared to control individuals $(0.7 \pm 1.2$ dots/field; $p<0.05$ vs FAD, $p<0.01$ vs CBD and $p<0.05$ vs PSP). Altogether, our data suggest that pathological tau is present in LC3positive structures in FAD, CBD and PSP human postmortem brain.

\section{Accumulation of lysosomal markers and impairment of lysosomal integrity in tauopathies}

The accumulation of autophagic vesicles in AD patients has been suggested to be a result of defective lysosomal clearance $[37,39,70]$. To investigate this hypothesis, we analysed the localisation and the integrity of lysosomes in frontal cortex of tauopathy patients. First, we analysed the protein level of the lysosomal-associated membrane protein 1 (LAMP1), a major lysosomal glycoprotein, by Western blot. We found significantly higher LAMP1 protein levels in frontal cortex from FAD (AU $0.42 \pm$ 0.09 ) and $\mathrm{CBD}$ (AU $0.38 \pm 0.03$ ) compared to control (AU $0.15 \pm 0.062, p<0.01$ vs FAD and $p<0.05$ vs CBD, Fig. 5a-b). Frozen brain tissue for Western blot analysis was not available from patients with PSP.

Next, we analysed the expression of Cathepsin D (Cat D), a major lysosomal hydrolase involved in the proteolytic degradation of proteins in lysosomes, by immunofluorescence. In frontal cortex from controls, we observed small perinuclear Cat D-positive punctate structures, corresponding to lysosomes (Fig. 5j, n, t, asterisks and Additional file 3: Figure S3). In contrast, the Cat D immunoreactivity was stronger in FAD, CBD and PSP patients compared to control individuals (Fig. 5g-i and Additional file 3: Figure S3). Two different patterns of Cat D immunoreactivity were observed: (1) a staining pattern of small vesicular structures, corresponding to lysosomes (Fig. 5q-s, asterisks) and (2) a 


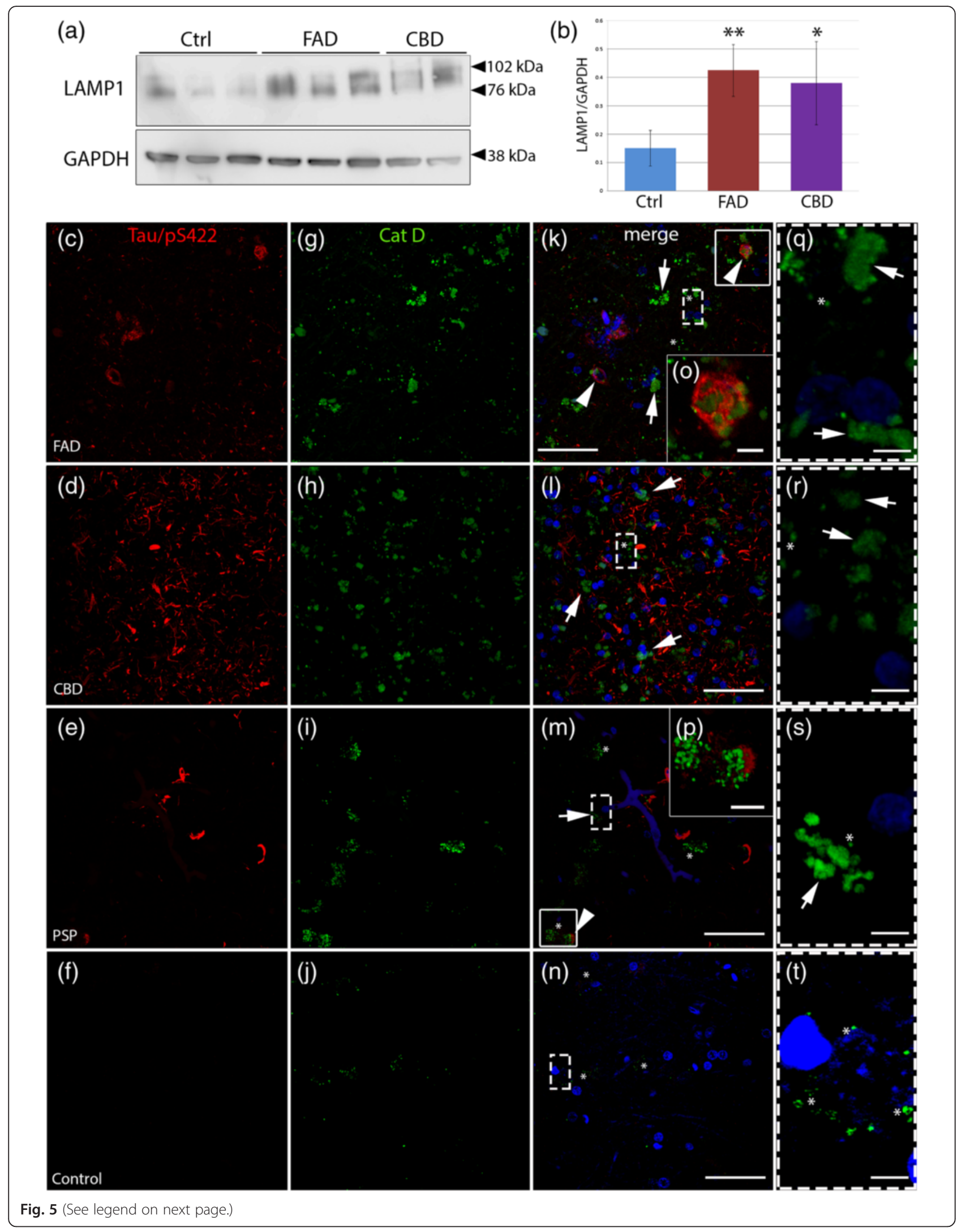




\section{(See figure on previous page.)}

Fig. 5 Accumulation of lysosomal markers and diffuse cytoplasmic Cat D immunoreactivity in tauopathies. a Western blot analysis of LAMP1 in human post-mortem brain (frontal cortex) extracts from control individuals ( $C t r l, n=3)$, early onset familial AD (FAD, $n=3)$ and CBD $(n=2)$. b LAMP1 levels quantified by densitometry and normalized to GAPDH in Ctrl, FAD and CBD. Results are shown as the mean value of arbitrary unit \pm S.D (mean of three independent experiments). c-t Double immunohistochemistry against hyperphosphorylated tau (Tau/pS422, red, c-f) and Cat D (HPA003001, Sigma-Aldrich) (green, $\mathbf{g - j}$ ) are shown by confocal analysis. $\mathbf{k}-\mathbf{n}$ Merged pictures with nuclear staining (DAPI, blue). In FAD, CBD and PSP cases, strong Cat D-immunoreactivity is present in the soma of the cells in frontal cortex compared to the control. $\mathbf{k}-\mathbf{m}$ (and high magnification $\mathbf{q}-\mathbf{s}$ ) Distinct vesicle-like structures (asterisks) and diffuse immunoreactivity (arrows) of Cat D throughout the cell bodies are shown in the pictures. In addition, in FAD and PSP, arrowheads in $\mathbf{k}$ and $\mathbf{m}$ and high magnification pictures (o-p) of boxed areas show hyperphosphorylated taupositive structures close to Cat D-positive structures. $\mathbf{t}$ Brain tissues from control individuals show few Cat D-positive vesicle structures (asterisks). Scale bars: $50 \mu \mathrm{m}$ (5 $\mu \mathrm{m}$ high magnification)

diffuse Cat D staining pattern showing a fluorescent signal throughout the cytoplasm (Fig. 5q-s, arrows, and Additional file 3: Figure S3), suggesting that Cat D could have leaked out of the lysosomes (reviewed in $[8,74,78])$. Taken together the Cat $\mathrm{D}$ results suggest that the lysosomal integrity may be impaired in all three patient groups.

Finally we analysed the colocalization of hyperphosphorylated tau with Cat D using double immunofluorescence. In all three patient groups, we found that Cat D-positive staining rarely colocalized with hyperphosphorylated tau and only a few cells were immunoreactive for both Tau/pS422 (red) and Cat D (green) (arrowheads and inset in FAD and PSP, Fig. 5k, m, o, p). This suggests that hyperphosphorylated tau is not localized to lysosomes which is in line with previous studies in sporadic $\mathrm{AD}$ patients $[5,15]$.

\section{Discussion}

Here we used human post-mortem brain tissue to demonstrate that abnormal accumulation of markers of the autophagy-lysosomal pathway is a common feature of different tauopathies. We found that patients with primary (CBD and PSP) and secondary (early onset FAD) tauopathies show an abnormal accumulation of the autophagy markers p62/SQSTM1 and LC3, and that both these markers colocalized with hyperphosphorylated tau. In FAD patients, hyperphosphorylated tau colocalized with LC3-positive structures and p62/SQSTM1 both in the soma and in neuritic threads, while in CBD and PSP patients, colocalization was found mainly in threads. Previous studies have demonstrated that hyperphosphorylated tau impairs microtubulebased axonal retrograde transport [27, 44, 46, 49], and that disruption of microtubule-based vesicle transport, either by the microtubule-depolymerizing drug vinblastine or by deleting the motor-protein dynein, results in a massive accumulation of autophagosomes in neurites $[7,28]$. Thus, it is tempting to speculate that the accumulation of hyperphosphorylated tau, p62/ SQSTM1 and LC3 in threads in the tauopathy patients are, at least partly, due to impaired axonal transport. It should be noted that in CBD and PSP patients, hyperphosphorylated tau is found both in neurons and in glial cells, and although the autophagy-lysosomal pathway has been demonstrated to be the main degradative pathway of tau in neurons, other mechanisms (including the ubiquitin-proteasome system) may be involved in degradation of tau in other cell types such as glia $[32,67,68]$.

Our analysis of lysosomal markers showed increased protein levels of LAMP1 and Cat D in FAD patients as well as in patients with primary tauopathies, suggesting a defective lysosomal clearance which is in line with previous reports in $\mathrm{AD}$ brain $[10,12,48,53,58]$. In contrast to the control cases where Cat D staining was typically punctate, indicating localization within lysosomes, we observed diffuse cytoplasmic Cat D immunoreactivity in the tauopathy patients. Diffuse Cat D staining in the soma is indicative of lysosomal membrane rupture [1], and can be induced by oxidative stress $[26,59]$. It has also been reported that tau can interact with lysosomal membranes and trigger lysosomal permeability in vitro [68] and that small tau fibrils can bind to lysosomal membranes resulting in lysosomal damage in a transgenic mouse model of AD [15]. Further studies are needed to determine if there is a leakage of Cat D into the cytoplasm in human tauopathies, but our data indicate that defective lysosomal integrity is prominent in FAD, CBD and PSP patients. It should be noted that the increased LAMP1 levels could be attributable to gliosis in the tauopathy patients $[5,11,13]$.

In recent years, several studies have indicated that (pharmacological) induction of autophagy can be beneficial for treatment of neurodegenerative diseases, and increased autophagy has been shown to ameliorate pathology in various disease models by enhancing the clearance of intracytoplasmic protein aggregates, including hyperphosphorylated tau [22, 66]. It is possible that an impaired retrograde transport could explain the observed accumulation of LC3-positive structures containing hyperphosphorylated tau in threads in tauopathy patients. On the other hand, our findings from the FAD cases suggest that hyperphosphorylated tau can be transported to 
the soma where we observed colocalization with LC3 and p62/SQSTM1.

\section{Conclusions}

In conclusion, our findings give support for an impairment of the autophagy-lysosomal system in patients with primary tauopathies as well as in familial AD caused by the Swedish APP mutation. Although the autophagosomallysosomal clearance pathway is compromised in all three tauopathies, our observations also indicate that the precise location of the impairment along this pathway is not necessarily the same. Thus, our study highlights the fact that therapeutic strategies targeting the autophagosomallysosomal pathway may need to be specifically tailored for different tauopathies.

\section{Ethical approval}

All procedures performed in studies involving human participants were in accordance with the ethical standards of the institutional and/or national research committee (the Regional Ethical Review Board in Stockholm, Sweden) and with the 1964 Helsinki declaration and its later amendments or comparable ethical standards.

\section{Additional files}

Additional file 1: Figure S1. Tau pathology observed with the Tau/pS422 antibody. a-f Immunofluorescence staining against hyperphosphorylated tau (Tau/pS422) in human brain samples. Intense immunoreactivity in FAD, CBD and PSP. a FAD cases show immunoreactivity in neurofibrillary tangles, pretangles, neuropil threads and dystrophic neurites. b-c CBD and PSP cases show immunoreactivity in neurons and glial cells. d-f High magnification of the boxed areas in a-c. Scale bars: $50 \mu \mathrm{m}$ (10 $\mu$ m high magnification) (TIF $3632 \mathrm{~kb}$ )

\section{Additional file 2: Figure S2. Colocalization between}

hyperphosphorylated tau (Tau/pS422) and LC3 in AD cases. a-d Double immunofluorescence staining against hyperphosphorylated tau (a) and LC3 (b) and merge (c) pictures in human brain samples show colocalization in neurons (NFT-like structures) in FAD samples. $d$ Superposition of confocal stacks. Scale bar: 10 m (TIF 25517 kb)

Additional file 3: Figure S3. Diffuse staining pattern of Cathepsin D (Cat D) (ab6313, Abcam) in tauopathies a-h Merged pictures show immunofluorescence staining of Cat D (green) and DAPI (nuclear marker, blue). a-c Diffuse immunoreactivity for Cat D in FAD, CBD and PSP patients (arrows) and distinct vesicle-like structures (asterisks). $\mathrm{d}$ and $\mathrm{h}$ Control samples show Cat D-positive vesicular-like structure (asterisks) and not diffuse staining. e-h High magnification of the boxed areas in a-d. Scale bars: $50 \mu \mathrm{m}$ (20 $\mu \mathrm{m}$ high magnification). (TIF $25515 \mathrm{~kb}$ )

\section{Competing interests}

LC and FG are under paid employment by the company F. Hoffmann-La Roche.

\section{Authors' contributions}

The project was designed by FG, LC, CG and AR. AP carried out the experimental work. The manuscript was prepared by AP, FG, LC, CG and AR. All authors read and approved the final manuscript.

\section{Acknowledgement}

AP was supported by the Roche Postdoctoral Fellowship Program. We thank Anna-Karin Lindström for excellent technical support, neuropathologist Inger Nennesmo for the clinical neuropathological characterization of the patients included in the study. We also thank Helena Karlström for guidance on the Western blot analyses and Sophia Schedin-Weiss for her technical guidance of the in situ PLA analyses.

\section{Funding}

This study was funded by the Roche Postdoc Fellowship program.

\section{Author details}

'Karolinska Institutet, Department NVS, Center for Alzheimer Research, Division for Neurogeriatrics, Karolinska Institutet, SE-141 57 Huddinge, Sweden. ${ }^{2}$ Roche Pharma Research and Early Development, NORD DTA, Roche Innovation Center Basel, Basel, Switzerland. ${ }^{3}$ Department Geriatric Medicine, Genet. Unit, Karolinska University Hospital, Stockholm, Sweden.

Received: 17 February 2016 Accepted: 18 February 2016

Published online: 02 March 2016

\section{References}

1. Aits $\mathrm{S}$, Jaattela M, Nylandsted J. Methods for the quantification of lysosomal membrane permeabilization: a hallmark of lysosomal cell death. Methods Cell Biol. 2015;126:261-85. doi:10.1016/bs.mcb.2014.10.032.

2. Babu JR, Geetha T, Wooten MW. Sequestosome 1/p62 shuttles polyubiquitinated tau for proteasomal degradation. J Neurochem. 2005;94:192-203. doi:10.1111/j.1471-4159.2005.03181.x.

3. Ballatore C, Lee VM, Trojanowski JQ. Tau-mediated neurodegeneration in Alzheimer's disease and related disorders. Nat Rev Neurosci. 2007;8:663-72. doi:10.1038/nrn2194

4. Bardag-Gorce F, Francis T, Nan L, Li J, He Lue Y, French BA, French SW. Modifications in P62 occur due to proteasome inhibition in alcoholic liver disease. Life Sci. 2005;77:2594-602. doi:10.1016/.Ifs.2005.04.020.

5. Barrachina M, Maes T, Buesa C, Ferrer I. Lysosome-associated membrane protein 1 (LAMP-1) in Alzheimer's disease. Neuropathol Appl Neurobiol. 2006;32:505-16. doi:10.1111/j.1365-2990.2006.00756.x

6. Bjorkoy G, Lamark T, Johansen T. p62/SQSTM1 - A missing link between protein aggregates and the autophagy machinery. Autophagy. 2006;2:138-9.

7. Boland B, Kumar A, Lee S, Platt FM, Wegiel J, Yu WH, Nixon RA. Autophagy induction and autophagosome clearance in neurons: relationship to autophagic pathology in Alzheimer's disease. J Neurosci. 2008;28:6926-37. doi:10.1523/JNEUROSCI.0800-08.2008.

8. Boya P, Kroemer G. Lysosomal membrane permeabilization in cell death. Oncogene. 2008;27:6434-51. doi:10.1038/onc.2008.310.

9. Caccamo A, Magri A, Medina DX, Wisely EV, Lopez-Aranda MF, Silva AJ, Oddo S. mTOR regulates tau phosphorylation and degradation: implications for Alzheimer's disease and other tauopathies. Aging Cell. 2013;12:370-80. doi:10.1111/acel.12057.

10. Cataldo AM, Barnett JL, Berman SA, Li J, Quarless S, Bursztajn S, Lippa C, Nixon RA. Gene expression and cellular content of cathepsin D in Alzheimer's disease brain: evidence for early up-regulation of the endosomal-lysosomal system. Neuron. 1995;14:671-80.

11. Cataldo AM, Barnett JL, Mann DM, Nixon RA. Colocalization of lysosomal hydrolase and beta-amyloid in diffuse plaques of the cerebellum and striatum in Alzheimer's disease and Down's syndrome. J Neuropathol Exp Neurol. 1996;55:704-15.

12. Cataldo AM, Hamilton DJ, Nixon RA. Lysosomal abnormalities in degenerating neurons link neuronal compromise to senile plaque development in Alzheimer disease. Brain Res. 1994;640:68-80.

13. Cataldo AM, Paskevich PA, Kominami E, Nixon RA. Lysosomal hydrolases of different classes are abnormally distributed in brains of patients with Alzheimer disease. Proc Natl Acad Sci U S A. 1991;88:10998-1002.

14. Chesser AS, Pritchard SM, Johnson GV. Tau clearance mechanisms and their possible role in the pathogenesis of Alzheimer disease. Front Neurol. 2013;4:122. doi:10.3389/fneur.2013.00122.

15. Collin L, Bohrmann B, Gopfert U, Oroszlan-Szovik K, Ozmen L, Gruninger F. Neuronal uptake of tau/pS422 antibody and reduced progression of tau pathology in a mouse model of Alzheimer's disease. Brain. 2014;137:2834-46. doi:10.1093/brain/awu213.

16. Dolan PJ, Johnson GV. A caspase cleaved form of tau is preferentially degraded through the autophagy pathway. J Biol Chem. 2010;285:21978-87. doi:10.1074/jbc.M110.110940.

17. Dunn Jr WA. Studies on the mechanisms of autophagy: formation of the autophagic vacuole. J Cell Biol. 1990;110:1923-33. 
18. Dunn Jr WA. Studies on the mechanisms of autophagy: maturation of the autophagic vacuole. J Cell Biol. 1990;110:1935-45.

19. Gentry EG, Henderson BW, Arrant AE, Gearing M, Feng Y, Riddle NC, Herskowitz JH. Rho Kinase Inhibition as a Therapeutic for Progressive Supranuclear Palsy and Corticobasal Degeneration. J Neurosci. 2016:36:1316-23. doi:10.1523/JNEUROSCI.2336-15.2016.

20. Grundke-lqbal I, lqbal K, Tung YC, Quinlan M, Wisniewski HM, Binder LI. Abnormal phosphorylation of the microtubule-associated protein tau (tau) in Alzheimer cytoskeletal pathology. Proc Natl Acad Sci U S A. 1986:83:4913-7.

21. Guillozet-Bongaarts AL, Cahill ME, Cryns VL, Reynolds MR, Berry RW, Binder LI. Pseudophosphorylation of tau at serine 422 inhibits caspase cleavage: in vitro evidence and implications for tangle formation in vivo. J Neurochem. 2006:97:1005-14. doi:10.1111/j.1471-4159.2006.03784.x.

22. Hara T, Nakamura K, Matsui M, Yamamoto A, Nakahara Y, Suzuki-Migishima R, Yokoyama M, Mishima K, Saito I, Okano H, Mizushima N. Suppression of basal autophagy in neural cells causes neurodegenerative disease in mice. Nature. 2006;441:885-9. doi:10.1038/nature04724.

23. He C, Klionsky DJ. Regulation mechanisms and signaling pathways of autophagy. Annu Rev Genet. 2009;43:67-93. doi:10.1146/annurev-genet102808-114910.

24. Hernandez F, Avila J. Tauopathies. Cell Mol Life Sci. 2007;64:2219-33. doi:10.1007/s00018-007-7220-x

25. Inoue K, Rispoli J, Kaphzan H, Klann E, Chen El, Kim J, Komatsu M, Abeliovich A. Macroautophagy deficiency mediates age-dependent neurodegeneration through a phospho-tau pathway. Mol Neurodegener. 2012;7:48. doi:10.1186/1750-1326-7-48.

26. Kagedal K, Johansson U, Ollinger K. The lysosomal protease cathepsin D mediates apoptosis induced by oxidative stress. FASEB J. 2001;15:1592-4.

27. Kim J, Choi IY, Michaelis ML, Lee P. Quantitative in vivo measurement of early axonal transport deficits in a triple transgenic mouse model of Alzheimer's disease using manganese-enhanced MRI. Neuroimage. 2011;56:1286-92. doi:10.1016/j.neuroimage.2011.02.039.

28. Kimura S, Noda T, Yoshimori T. Dynein-dependent movement of autophagosomes mediates efficient encounters with lysosomes. Cell Struct Funct. 2008;33:109-22.

29. Klionsky DJ, Abdalla FC, Abeliovich H, Abraham RT, Acevedo-Arozena A, Adeli K, et al. Guidelines for the use and interpretation of assays for monitoring autophagy. Autophagy. 2012;8:445-544.

30. Klionsky DJ, Emr SD. Autophagy as a regulated pathway of cellular degradation. Science. 2000;290:1717-21.

31. Komatsu M, Waguri S, Chiba T, Murata S, Iwata J, Tanida I, Ueno T, Koike M, Uchiyama Y, Kominami E, Tanaka K. Loss of autophagy in the central nervous system causes neurodegeneration in mice. Nature. 2006;441:880-4. doi:10.1038/nature04723.

32. Kruger U, Wang Y, Kumar S, Mandelkow EM. Autophagic degradation of tau in primary neurons and its enhancement by trehalose. Neurobiol Aging. 2012;33:2291-305. doi:10.1016/j.neurobiolaging.2011.11.009.

33. Kuusisto E, Kauppinen T, Alafuzoff I. Use of p62/SQSTM1 antibodies for neuropathological diagnosis. Neuropathol Appl Neurobiol. 2008;34:169-80. doi:10.1111/j.1365-2990.2007.00884.x.

34. Kuusisto E, Salminen A, Alafuzoff I. Ubiquitin-binding protein p62 is present in neuronal and glial inclusions in human tauopathies and synucleinopathies. Neuroreport. 2001;12:2085-90.

35. Kuusisto E, Salminen A, Alafuzoff I. Early accumulation of p62 in neurofibrillary tangles in Alzheimer's disease: possible role in tangle formation. Neuropathol Appl Neurobiol. 2002;28:228-37.

36. Lawrence BP, Brown WJ. Autophagic vacuoles rapidly fuse with pre-existing lysosomes in cultured hepatocytes. J Cell Sci. 1992;102(Pt 3):515-26.

37. Lee JH, Yu WH, Kumar A, Lee S, Mohan PS, Peterhoff CM, Wolfe DM, Martinez-Vicente M, Massey AC, Sovak G, Uchiyama Y, Westaway D, Cuervo AM, Nixon RA. Lysosomal proteolysis and autophagy require presenilin 1 and are disrupted by Alzheimer-related PS1 mutations. Cell. 2010;141:1146-58. doi:10.1016/j.cell.2010.05.008.

38. Lee MJ, Lee JH, Rubinsztein DC. Tau degradation: the ubiquitin-proteasome system versus the autophagy-lysosome system. Prog Neurobiol. 2013;105:49-59. doi:10.1016/j.pneurobio.2013.03.001.

39. Lee S, Sato Y, Nixon RA. Lysosomal proteolysis inhibition selectively disrupts axonal transport of degradative organelles and causes an Alzheimer's-like axonal dystrophy. J Neurosci. 2011;31:7817-30. doi:10.1523/JNEUROSCI.6412-10.2011.
40. Leyk J, Goldbaum O, Noack M, Richter-Landsberg C. Inhibition of HDAC6 modifies tau inclusion body formation and impairs autophagic clearance. J Mol Neurosci. 2015;55:1031-46. doi:10.1007/s12031-014-0460-y.

41. Lin WL, Lewis J, Yen SH, Hutton M, Dickson DW. Ultrastructural neuronal pathology in transgenic mice expressing mutant (P301L) human tau. J Neurocytol. 2003:32:1091-105. doi:10.1023/B:NEUR.0000021904.61387.95.

42. Lipinski MM, Zheng B, Lu T, Yan Z, Py BF, Ng A, Xavier RJ, Li C, Yankner BA, Scherzer CR, Yuan J. Genome-wide analysis reveals mechanisms modulating autophagy in normal brain aging and in Alzheimer's disease. Proc Natl Acad Sci U S A. 2010;107:14164-9. doi:10.1073/pnas.1009485107.

43. Liu Z, Li T, Li P, Wei N, Zhao Z, Liang H, Ji X, Chen W, Xue M, Wei J. The ambiguous relationship of oxidative stress, Tau hyperphosphorylation, and autophagy dysfunction in Alzheimer's disease. Oxid Med Cell Longev. 2015;2015:352723. doi:10.1155/2015/352723.

44. Majid T, Ali YO, Venkitaramani DV, Jang MK, Lu HC, Pautler RG. In vivo axonal transport deficits in a mouse model of fronto-temporal dementia. Neuroimage Clin. 2014:4:711-7. doi:10.1016/j.nicl.2014.02.005.

45. Mandelkow EM, Mandelkow E. Tau in Alzheimer's disease. Trends Cell Biol. 1998;8:425-7.

46. Morfini G, Pigino G, Beffert U, Busciglio J, Brady ST. Fast axonal transport misregulation and Alzheimer's disease. Neuromolecular Med. 2002;2:89-99. doi:10.1385/NMM:2:2:089

47. Morris M, Knudsen GM, Maeda S, Trinidad JC, loanoviciu A, Burlingame AL, Mucke L. Tau post-translational modifications in wild-type and human amyloid precursor protein transgenic mice. Nat Neurosci. 2015;18:1183-9. doi:10.1038/nn.4067.

48. Nakamura Y, Takeda M, Suzuki H, Hattori H, Tada K, Hariguchi S, Hashimoto S, Nishimura T. Abnormal distribution of cathepsins in the brain of patients with Alzheimer's disease. Neurosci Lett. 1991;130:195-8.

49. Niewiadomska G, Baksalerska-Pazera M, Riedel G. Altered cellular distribution of phospho-tau proteins coincides with impaired retrograde axonal transport in neurons of aged rats. Ann N Y Acad Sci. 2005;1048:287-95. doi:10.1196/annals.1342.026.

50. Nixon RA. Autophagy in neurodegenerative disease: friend, foe or turncoat? Trends Neurosci. 2006;29:528-35. doi:10.1016/j.tins.2006.07.003.

51. Nixon RA. Autophagy, amyloidogenesis and Alzheimer disease. J Cell Sci. 2007;120:4081-91. doi:10.1242/jcs.019265.

52. Nixon RA. The role of autophagy in neurodegenerative disease. Nat Med. 2013;19:983-97. doi:10.1038/nm.3232.

53. Nixon RA, Cataldo AM, Paskevich PA, Hamilton DJ, Wheelock TR, KanaleyAndrews $\mathrm{L}$. The lysosomal system in neurons. Involvement at multiple stages of Alzheimer's disease pathogenesis. Ann N Y Acad Sci. 1992;674:65-88.

54. Nixon RA, Wegiel J, Kumar A, Yu WH, Peterhoff C, Cataldo A, Cuervo AM. Extensive involvement of autophagy in Alzheimer disease: an immunoelectron microscopy study. J Neuropathol Exp Neurol. 2005;64:113-22.

55. Orr ME, Oddo S. Autophagic/lysosomal dysfunction in Alzheimer's disease. Alzheimers Res Ther. 2013;5:53. doi:10.1186/alzrt217.

56. Ozcelik S, Fraser G, Castets P, Schaeffer V, Skachokova Z, Breu K, Clavaguera F, Sinnreich M, Kappos L, Goedert M, Tolnay M, Winkler DT. Rapamycin attenuates the progression of tau pathology in P301S tau transgenic mice. PLoS ONE. 2013;8:e62459. doi:10.1371/journal.pone.0062459.

57. Pankiv S, Clausen TH, Lamark T, Brech A, Bruun JA, Outzen H, Overvatn A, Bjorkoy G, Johansen T. p62/SQSTM1 binds directly to Atg8/LC3 to facilitate degradation of ubiquitinated protein aggregates by autophagy. J Biol Chem. 2007;282:24131-45. doi:10.1074/jbc.M702824200.

58. Perez SE, He B, Nadeem M, Wuu J, Ginsberg SD, Ikonomovic MD, Mufson EJ. Hippocampal endosomal, lysosomal, and autophagic dysregulation in mild cognitive impairment: correlation with abeta and tau pathology. J Neuropathol Exp Neurol. 2015;74:345-58. doi:10.1097/NEN.0000000000000179.

59. Roberg K. Relocalization of cathepsin D and cytochrome c early in apoptosis revealed by immunoelectron microscopy. Lab Invest. 2001;81:149-58.

60. Sato-Harada R, Okabe S, Umeyama T, Kanai Y, Hirokawa N. Microtubuleassociated proteins regulate microtubule function as the track for intracellular membrane organelle transports. Cell Struct Funct. 1996;21:283-95.

61. Schaeffer V, Lavenir I, Ozcelik S, Tolnay M, Winkler DT, Goedert M. Stimulation of autophagy reduces neurodegeneration in a mouse model of human tauopathy. Brain. 2012;135:2169-77. doi:10.1093/brain/aws143.

62. Schedin-Weiss S, Inoue M, Teranishi Y, Yamamoto NG, Karlstrom H, Winblad $B$, Tjernberg LO. Visualizing active enzyme complexes using a photoreactive inhibitor for proximity ligation-application on gamma-secretase. PLOS ONE. 2013;8:e63962. doi:10.1371/journal.pone.0063962. 
63. Seibenhener ML, Babu JR, Geetha T, Wong HC, Krishna NR, Wooten MW. Sequestosome 1/p62 is a polyubiquitin chain binding protein involved in ubiquitin proteasome degradation. Mol Cell Biol. 2004;24:8055-68. doi:10. 1128/MCB.24.18.8055-8068.2004.

64. Soderberg O, Gullberg M, Jarvius M, Ridderstrale K, Leuchowius KJ, Jarvius J, Wester K, Hydbring P, Bahram F, Larsson LG, Landegren U. Direct observation of individual endogenous protein complexes in situ by proximity ligation. Nat Methods. 2006;3:995-1000. doi:10.1038/nmeth947.

65. Tang Z, Bereczki E, Zhang H, Wang S, Li C, Ji X, Branca RM, Lehtio J, Guan Z, Filipcik P, Xu S, Winblad B, Pei JJ. Mammalian target of rapamycin (mTor) mediates tau protein dyshomeostasis: implication for Alzheimer disease. J Biol Chem. 2013;288:15556-70. doi:10.1074/jbc.M112.435123.

66. Voss K, Koren 3rd J, Dickey CA. The earliest tau dysfunction in Alzheimer's disease? Tau phosphorylated at $\$ 422$ as a toxic seed. Am J Pathol. 2011;179:2148-51. doi:10.1016/j.ajpath.2011.08.020.

67. Wang Y, Mandelkow E. Degradation of tau protein by autophagy and proteasomal pathways. Biochem Soc Trans. 2012;40:644-52. doi:10.1042/ BST20120071.

68. Wang Y, Martinez-Vicente M, Kruger U, Kaushik S, Wong E, Mandelkow EM, Cuervo AM, Mandelkow E. Tau fragmentation, aggregation and clearance: the dual role of lysosomal processing. Hum Mol Genet. 2009;18:4153-70. doi:10.1093/hmg/ddp367.

69. Weingarten MD, Lockwood AH, Hwo SY, Kirschner MW. A protein factor essential for microtubule assembly. Proc Natl Acad Sci U S A. 1975;72:1858-62.

70. Wolfe DM, Lee JH, Kumar A, Lee S, Orenstein SJ, Nixon RA. Autophagy failure in Alzheimer's disease and the role of defective lysosomal acidification. Eur J Neurosci. 2013;37:1949-61. doi:10.1111/ejn.12169.

71. Wong MB, Goodwin J, Norazit A, Meedeniya AC, Richter-Landsberg C, Gai WP, Pountney DL. SUMO-1 is associated with a subset of lysosomes in glial protein aggregate diseases. Neurotox Res. 2013;23:1-21. doi:10.1007/s12640012-9358-z.

72. Xie Z, Klionsky DJ. Autophagosome formation: core machinery and adaptations. Nat Cell Biol. 2007;9:1102-9. doi:10.1038/ncb1007-1102.

73. Yamamoto A, Tagawa Y, Yoshimori T, Moriyama Y, Masaki R, Tashiro Y. Bafilomycin A1 prevents maturation of autophagic vacuoles by inhibiting fusion between autophagosomes and lysosomes in rat hepatoma cell line, H-4-II-E cells. Cell Struct Funct. 1998;23:33-42.

74. Yamashima T, Oikawa S. The role of lysosomal rupture in neuronal death. Prog Neurobiol. 2009:89:343-58. doi:10.1016/j.pneurobio.2009.09.003.

75. Yang Z, Klionsky DJ. Eaten alive: a history of macroautophagy. Nat Cell Biol. 2010;12:814-22. doi:10.1038/ncb0910-814.

76. Yang Z, Klionsky DJ. Mammalian autophagy: core molecular machinery and signaling regulation. Curr Opin Cell Biol. 2010;22:124-31. doi:10.1016/j.ceb. 2009.11.014.

77. Yu WH, Kumar A, Peterhoff C, Shapiro Kulnane L, Uchiyama Y, Lamb BT, Cuervo AM, Nixon RA. Autophagic vacuoles are enriched in amyloid precursor protein-secretase activities: implications for beta-amyloid peptide over-production and localization in Alzheimer's disease. Int J Biochem Cell Biol. 2004;36:2531-40. doi:10.1016/j.biocel.2004.05.010.

78. Zhang L, Sheng R, Qin Z. The lysosome and neurodegenerative diseases. Acta Biochim Biophys Sin (Shanghai). 2009;41:437-45.

\section{Submit your next manuscript to BioMed Central and we will help you at every step:}

- We accept pre-submission inquiries

- Our selector tool helps you to find the most relevant journal

- We provide round the clock customer support

- Convenient online submission

- Thorough peer review

- Inclusion in PubMed and all major indexing services

- Maximum visibility for your research

Submit your manuscript at www.biomedcentral.com/submit
Biomed Central 